\title{
Doxorubicin Induces Apoptosis in H9c2 Cardiomyocytes: Role of Overexpressed Eukaryotic Translation Initiation Factor 5A
}

\author{
Xiao Tan, ${ }^{\#}$ Di-bin Wang, ${ }^{\#}$ Xiang Lu, Hui Wei, Rong Zhu, Shu-shu Zhu, Hai Jiang, and \\ Zhi-jian YANG* \\ Department of Cardiology, Second Clinical Medical School of Nanjing Medical University; Nanjing 210011, P. R. China. \\ Received May 21, 2010; accepted July 5, 2010; published online July 30, 2010
}

\begin{abstract}
The cardiotoxicity of doxorubicin limits its clinical use in the treatment of a variety of solid tumors and malignant hematologic disease. Although the mechanism by which it causes cardiac injury is not yet known, apoptosis has been regarded as one of mechanisms underlying the cardiotoxic effects of doxorubicin. Eukaryotic translation initiation factor 5A (eIF5A) is a ubiquitously expressed multifunctional protein that interacts with a range of ligands and is implicated in cell signaling. However, there has been no direct evidence for the critical involvement of eIF5A in doxorubicin-induced apoptosis. Overexpression of eIF5A induced by doxorubicin in cardiomyocyte leads to growth perturbation along with initiation of apoptosis. Overexpression of eIF5A results in a gradual increase in reactive oxygen species (ROS) generation. This mitochondrial dysfunction is due to a gradual increase in ROS generation in eIF5A-overexpressing $\mathrm{H} 9 \mathrm{c} 2$ cells. Along with ROS generation, increased $\mathrm{Ca}^{2+}$ influx in mitochondria leads to loss of the mitochondrial transmembrane potential, release of cytochrome-c, and caspase activation. However, small interfering RNA (siRNA)-mediated suppression of eIF5A results in inhibition of apoptosis. Interestingly, upon overexpression of eIF5A induced by doxorubicin, cell apoptosis was shown to be significantly inhibited when cells were treated with SB202190 (p38 mitogen-activated protein kinase inhibitor) and SP600125 (anti-c-Jun N-terminal kinase inhibitor) for $18 \mathrm{~h}$. The reduction in oxidant generation and reduction in the apoptotic cell population were the results of the disruption of eIF5A expression, corroborating the hypothesis that excess ROS generation with overexpression of eIF5A induced by doxorubicin leads to apoptosis due to the accumulation of eIF5A.
\end{abstract}

Key words cardiotoxicity; doxorubicin; apoptosis; eukaryotic translation initiation factor 5A

Doxorubicin is an effective, widely used chemotherapeutic agent in the treatment of a variety of solid tumors and malignant hematologic disease. ${ }^{1)}$ However, cardiac toxicity, including the development of cardiomyopathy and clinical congestive failure, restricts the clinical use of the drug. ${ }^{2)}$ Therefore an understanding of the mechanisms that underlie doxorubicin-induced cardiac injury is crucial for the development of strategies to inhibit its cardiotoxic action. Studies have shown that treatment with doxorubicin results in transactivation into the nucleus to induce the expression of genes associated with cell arrest and apoptosis in the heart. ${ }^{3,4)} \mathrm{A}$ molecular mechanism leading to doxorubicin-induced cardiomyopathy has been proposed to account for increasing oxidant production, altered calcium handling, and mitochondrial injury. ${ }^{5-7)}$ This is probably reflected by the profound cardiomyocyte damage in certain states, which interferes with mitochondrial function.

Eukaryotic translation initiation factor 5A (eIF5A) is a highly conserved essential protein, which is the only protein that contains the unusual spermidine-derived amino acid residue hypusine. ${ }^{8)}$ In several mammalian cell types, eIF5A is an RNA-binding protein, which induced apoptosis via activation of the intrinsic mitochondrial pathway. ${ }^{9)}$ In humans, eIF5A is encoded by two genes, eIF5A1 and eIF5A2. eIF5A1 mRNA and protein appear to be present in all human tissues and cell types. However, eIF5A2 exhibits restricted expression in normal healthy tissue and is overexpressed in some cancers and cancer cell lines, prompting the suggestion that it may be an oncogene. However, data accumulated over the past 20 years have firmly established that the list of biologic responses mediated by eIF5A is extensive. Examples of such responses include phagocytosis and the uptake of apoptotic cells. $^{10)}$

In this study, we attempted to determine whether that the increase in p38 mitogen-activated protein kinase inhibitor and anti-c-Jun N-terminal kinase inhibitor activities of cardiomyocytes in response to eIF5A overexpression is associated with doxorubicin-induced apoptosis through the mitochondrial pathway.

\section{MATERIALS AND METHODS}

Reagents The cardiac muscle cell line $\mathrm{H} 9 \mathrm{c} 2$ was obtained from the China Type Culture Collection. Small interference RNA (siRNA) was synthesized by Wuhan Genesil Biotechnology Co., Ltd. (Wuhan, China). Lipofectin was purchased from Invitrogen (Carlsbad, CA, U.S.A.). The phototopeHorseradish Peroxidase (HRP) Western Blot Detection System, including anti-mouse immunoglobulin $\mathrm{G}$ (IgG), HRPlinked antibody, biotinylated protein ladder, 20X LumiGLO Reagent, and 20X Peroxide, were purchased from Cell Signaling Technology (Beverly, MA, U.S.A.). SB202190 (p38 mitogen-activated protein kinase (MAPK) inhibitor) and SP600125 (JNK inhibitor) were obtained from SigmaAldrich Inc. 5-(6)-Chloromethyl-2', 7'-dichlorodihy-drofluorescein diacetate $\left(\mathrm{H}_{2} \mathrm{DCFDA}\right)$ was the product of Molecular Probes (U.S.A.). The annexin V-fluorescein isothiocyanate (FITC)/propidium iodide flow cytometry assay kit was purchased from Invitrogen. Anti-p38 MAPK, anti-phospho-p38 MAPK, anti-c-Jun N-terminal kinase (JNK), antiphosphoJNK, anti-eIF5A, and caspase-9 antibodies were products of Cell Signaling Technology. Anti-cytochrome-c was purchased from BD Pharmingen, and horseradish peroxidase-conjugated secondary antibodies were obtained from Pierce. Cell 
culture supplies were purchased from Gibco/Life Technologies Inc. Metformin (promoter of mitochondrial biosynthesis, ${ }^{11)}$ positive control) was from Invitrogen. Unless otherwise specified, all other reagents were of analytical grade.

Cardiac Muscle Cell Line H9c2 Culture and DNA Transfection The cardiac muscle cell line H9c2 was maintained at $37^{\circ} \mathrm{C}$ in a $5 \% \mathrm{CO}_{2}$ incubator, in Dulbecco's modified Eagle's medium (DMEM) supplemented with $10 \%$ fetal calf serum (FCS), penicillin $100 \mathrm{U} / \mathrm{ml}$, streptomycin $100 \mu \mathrm{g} /$ $\mathrm{ml}$, and $10 \%$ unnecessary amino acid. Cells used in experiments were from 5 to 7 passages. Lipofectin- and oligofectamine-transfection of eIF5A siRNA was performed according to the vendor's protocol. Briefly, to cardiomyocytes in one well of a six-well plate, $500 \mathrm{pmol}$ of each expression vector and lipofectin $10 \mu \mathrm{l}$ were diluted in $750 \mu \mathrm{l}$ of OptiMEM (Life Technologies, U.S.A.). After preincubation of the lipofectin solution for $45 \mathrm{~min}$ at $37^{\circ} \mathrm{C}$, both solutions were mixed and incubated for an additional $15 \mathrm{~min}$ at room temperature. The lipofectin/siRNA mixture was subsequently overlaid onto the cells and incubated for $2 \mathrm{~h}$. Finally, $1 \mathrm{ml}$ of growth medium $(20 \%$ FCS $)$ per well was added for further cultivation of the H9c2 cells. Reporter gene activities were normalized to total protein, and all results represent the average of triplicate experiments.

Construction of eIF5A siRNA-Expressing Plasmid Vector The complementary oligonucleotides encoded a hairpin structure with a 19-mer stem derived from the target site. In this experiment, the targeted siRNA sequences for eIF5A were 5'-AAC GGA ATG ACT TCC AGC TGA-3'. Two complementary domains were separated by a 9-bp loop sequence. Near the $3^{\prime}$ end of the siRNA template was a 6-nucleotide poly(T) tract recognized as an RNA polIII termination signal. The $5^{\prime}$ end of the two oligonucleotides was BamHI with an overhanging HindIII restriction site. Using green fluorescent protein (GFP) as the reporter gene, the vectors of the eIF5A siRNA-expressing plasmid were constructed with pGenesil-1 as the vector backbone. The siRNA synthesized and annealed was ligated into the BamHI and HindIII site of the pGenesil-1 expression vector. At the same time, we chose an unrelated gene siRNA as a negative control.

Western Blot Analysis After specific treatments, H9c2 cells were incubated in lysis buffer containing Tris-HCl 20 $\mathrm{mm}$ ( $\mathrm{pH} 7.5), 1 \%$ Triton X-100, ethylene diamine tetracetic acid (EDTA) $1 \mathrm{~mm}, \mathrm{NaCl} 150 \mathrm{~mm}, 10 \%$ glycerol, $\mathrm{Na}_{3} \mathrm{VO}_{4}$ $1 \mathrm{~mm}$, NaF $50 \mathrm{~mm}$, phenylmethyl sulfonylfluoride (PMSF) $1 \mathrm{~mm}$ and protease inhibitor mixtures for $20 \mathrm{~min}$ on ice. After insoluble debris was precipitated by centrifugation at $13000 \times \boldsymbol{g}$ at $4{ }^{\circ} \mathrm{C}$ for $15 \mathrm{~min}$, the supernatants were collected. An equal amount of protein per sample $(15 \mu \mathrm{g})$ was resolved on $10 \%$ sodium dodecyl sulfate polyacrylamide gel electrophoresis (SDS-PAGE) and transferred onto a polyvinylidene fluoride (PVDF) membrane. The transferred membranes were blocked for $1 \mathrm{~h}$ in $5 \%$ nonfat milk in phosphate-buffered saline (PBS) containing $0.05 \%$ Tween 20 and incubated with appropriate primary antibodies and HRP-conjugated secondary antibodies. The immune complexes were detected with a SuperSignal West Pico Chemiluminescent substrate kit (Pierce).

Separation of Cytosolic and Mitochondrial Fractions for Cytochrome- $c$ Release After specific treatments, H9c2 cells were removed from the incubation plates $\left(6 \times 10^{6}\right.$ cells/100-mm dish). To separate the cytosolic and mitochondrial fractions, protein was extracted and differentially centrifuged. The activities of lactate dehydrogenase and citrate synthase in both fractions were measured to determine the cross-contamination between the cytosolic and mitochondrial fractions. The results demonstrated that cross-contamination between the cytosolic and mitochondrial fractions of $\mathrm{H} 9 \mathrm{c} 2$ cells were negligible. Then, cytochrome- $c$ in cytosolic and mitochondrial protein as detected using Western blot analysis.

Assay of Intracellular Reactive Oxygen Species (ROS) in $\mathrm{H} 9 \mathrm{c} 2$ Cells The production of intracellular $\mathrm{H}_{2} \mathrm{O}_{2}$ was based on the fluorescence of $\mathrm{H}_{2}$ DCFDA $(10 \mu \mathrm{M})$. Intracellular oxidants cause $\mathrm{H}_{2}$ DCFDA oxidation, yielding the fluorescent product 2,7-dichlorofluorescein (DCF). The assay was performed with fluorescence microscopy at an excitation wavelength of $488 \mathrm{~nm}$ and emission at $530 \mathrm{~nm}$. H9c2 cells were plated on Petri dishes and incubated with $\mathrm{H}_{2}$ DCFDA under various conditions for $10 \mathrm{~min}$ in the dark. The media were then removed, and $\mathrm{H} 9 \mathrm{c} 2$ cells were lysed with radio immunoprecipitation assay (RIPA) buffer ${ }^{12)}$ under ice-cold condition as rapidly as possible in a hypoxic environment and then centrifuged to remove debris. In the supernatant, the fluorescence was detected using a spectrofluorometer with a slit width of $5 \mathrm{~nm}$ (data were normalized to values obtained from normoxic untreated controls). The experiments were repeated at least 10 times, and for each experiment fluorometric measurements were performed in 10 different sets and expressed as the fold increase in fluorescence with respect to the experimental control by subtracting basal fluorescence.

Measurement of Intracellular $\mathrm{Ca}_{2}^{+}$Concentration The level of intracellular $\mathrm{Ca}_{2}^{+}$was quantified by fluorescence with Fluo-4 AM. H9c2 cells were treated under various conditions for the indicated times, and the treated cells were washed with ice-cold PBS. The cells were resuspended in $1 \mathrm{ml}$ of PBS and incubated with $5 \mathrm{ml}$ of Fluo-4 AM $1 \mathrm{~mm}$ for $1 \mathrm{~h}$. The fluorescence intensity of the intracellular $\mathrm{Ca}_{2}^{+}$concentration was measured with a Beckman Coulter Paradigm detection platform at an excitation wavelength of $485 \mathrm{~nm}$ and emission at $530 \mathrm{~nm}$. Results a shown as the increase in fluorescence intensity per microgram of proteins compared with the control group.

Measurement of Mitochondrial Membrane Potential The mitochondrial membrane potential $\left(\Delta \psi_{\mathrm{m}}\right)$ was estimated using a JC-1 probe according to the previously reported method $^{13)}$ with slight modifications. The cationic mitochondria-specific fluorescent dye was used to detect the loss of $\Delta \psi_{\mathrm{m}}$ following the induced apoptosis. The dye accumulates in mitochondria with increasing $\Delta \psi_{\mathrm{m}}$ and ATP-generating capacity and presents in monomeric condition at an excitation wavelength of $485 \mathrm{~nm}$ (at lower concentration) and emission at $530 \mathrm{~nm}$ (at higher concentration). Briefly, H9c2 cells treated under various conditions after $60 \mathrm{~h}$ of culture were washed with serum-free medium and incubated with JC-1 $10 \mu \mathrm{M}$ at $37^{\circ} \mathrm{C}$, then washed and resuspended with $10 \%$ serum containing medium, and measured at the two different wavelengths. The data reported are results after 10 repetitions of the experiments.

Detection of Apoptotic Cells Annexin V/propidium iodide staining was carried out according to the manufacturer's protocol (annexin V-FITC kit from Invitrogen). After treatment for the indicated times, H9c2 cells were washed with 
cold PBS and resuspended with binding buffer ( $N$-2-hydroxyethylpiperazine- $N^{\prime}$-2-ethanesulfonic acid (HEPES) $10 \mathrm{~mm}$, $\mathrm{pH} 7.4, \mathrm{NaCl} 140 \mathrm{~mm}, \mathrm{CaCl}_{2} 2.5 \mathrm{~mm}$ ) before transferring $1 \times$ $10^{5}$ cells to a $5-\mathrm{ml}$ tube. Then $5 \mathrm{ml}$ of annexin V-FITC and $5 \mathrm{ml}$ of propidium iodide were added, and the cells were incubated for $15 \mathrm{~min}$ in the dark. Binding buffer $(400 \mathrm{ml})$ was then added to each tube, and the DNA content of the cells was analyzed using a FACScan (Becton Dickinson). Apoptotic cells were quantitated by the percentage of cells with a sub-G1 DNA content.

Statistical Analysis Most results are presented as mean \pm S.D. Differences between various data sets were tested for significance using Student's $t$-test and a $p$ value of less than 0.05 was considered significant.

\section{RESULTS}

Expression of eIF5A Protein Induced by Doxorubicin in H9c2 Cells In this experiment, a suitable concentration of doxorubicin was investigated using the MTS cytotoxicity test (data not shown), and a dose-dependent potentiation of apoptosis was achieved. To investigate the effects of doxorubicin on the production of eIF5A protein, the expression of eIF5A in $\mathrm{H} 9 \mathrm{c} 2$ cells treated $(72 \mathrm{~h})$ with doxorubicin $(2 \mu \mathrm{M})$ was assessed (Fig. 1). The expression of eIF5A protein was significantly increased in $\mathrm{H} 9 \mathrm{c} 2$ cells treated with doxorubicin compared with controls. This finding suggests that eIF5A may play an important role in the survival of doxorubicintreated $\mathrm{H} 9 \mathrm{c} 2$ cells.

Doxorubicin Induces H9c2 Cell Apoptosis via eIF5A To investigate the role of eIF5A in doxorubicin-induced $\mathrm{H} 9 \mathrm{c} 2$ cell apoptosis, we used siRNA to inhibit the expression of eIF5A. In this experiment, H9c2 cells were transfected with eIF5A siRNA, negative siRNA, and empty siRNA. The expression of eIF5A was significantly decreased after transfection with eIF5A siRNA as compared with the PBS group during the experimental period. The negative siRNA and empty siRNA groups had no apparent change in eIF5A protein as compared with the PBS group. As shown in Fig. 2A, the optimal siRNA that inhibited the epression of the target genes as eIF5A siRNA. To study the functional role of eIF5A in doxorubicin-induced $\mathrm{H} 9 \mathrm{c} 2$ cell apoptosis, we treated $\mathrm{H} 9 \mathrm{c} 2$ cells with doxorubicin+eIF5A siRNA, doxorubicin+empty vector, doxorubicin + metformin (promoter of mitochondrial biosynthesis, positive control), and doxorubicin alone (control). As shown in Fig. 2B, FACS analysis revealed that the number of cells in the sub-G1 region increased after treatment with doxorubicin+empty vector and doxorubicin alone in $\mathrm{H} 9 \mathrm{c} 2$ cells. Under these conditions, inhibition of eIF5A markedly reduced the number of sub-G1 cells in the doxorubicin+eIF5A siRNA group. This inhibition of apoptosis was 5-fold higher than in the doxorubicin alone-treated $\mathrm{H} 9 \mathrm{c} 2$ cells. Meanwhile, to evaluate caspase-9 activation, the presence of the $17-\mathrm{kDa}$ caspase-9 fragment was examined in H9c2 cells by treating cells with doxorubicin+eIF5A siRNA, doxorubicin+empty vector, doxorubicin+metformin and doxorubicin alone (Fig. 2C). Overexpression of eIF5A induced an increase in the $17-\mathrm{kDa}$ fragment during $18-\mathrm{h}$ culture in the doxorubicin + empty vector group and doxorubicin alone group, whereas inhibition of eIF5A inhibited the production of caspase- 9 in the doxorubicin+eIF5A siRNA group
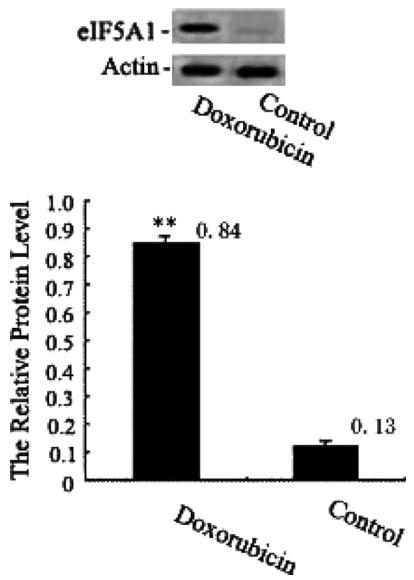

Fig. 1. Expression of eIF5A Protein in H9c2 Cell

The expression of eIF5A in H9c2 cells treated $(72 \mathrm{~h})$ with doxorubicin $(2 \mu \mathrm{M})$ was assessed in this experiment. The expression of eIF5A protein was measured by using Western blotting. The graph shows the relative eIF5A protein levels normalized to $\beta$ actin. Results shown are mean \pm S.D. of three independent transfections $(n=3)$, each conducted in triplicate. $* * p<0.01$ versus control group.

and doxorubicin + metformin group. Because metformin can promote mitochondrial biosynthesis, the production of caspase- 9 markedly decreased. This finding indicates that eIF5A may induce the mitochondrial pathway of $\mathrm{H} 9 \mathrm{c} 2$ cell apoptosis.

Doxorubicin Induced an Increase in $\mathrm{Ca}^{2+}$ and $\mathrm{ROS}$ Generation in H9c2 Cells via eIF5A The levels of $\mathrm{Ca}^{2+}$ and ROS in $\mathrm{H} 9 \mathrm{c} 2$ cells cultured with doxorubicin+eIF5A siRNA, doxorubicin +empty vector, doxorubicin + metformin and doxorubicin alone were evaluated. Cytosolic $\mathrm{Ca}^{2+}$ determined with a fluorescent enzyme-linked immunosorbent assay (ELISA) reader revealed a notable elevation in $\left[\mathrm{Ca}^{2+}\right]_{\mathrm{i}}$ after culture with doxorubicin+empty vector and doxorubicin alone (Fig. 3A). The levels of ROS in the doxorubicin+eIF5A siRNA, doxorubicin+empty vector, doxorubicin + metformin and doxorubicin alone-groups of $\mathrm{H} 9 \mathrm{c} 2$ cells were evaluated. Maximal ROS generation determined with the fluorescent ELISA reader was noted after doxorubicin+eIF5A siRNA, doxorubicin+empty vector, doxorubicin + metformin and doxorubicin-alone treatment for $1 \mathrm{~h}$, and then the ROS level in the doxorubicin+empty vector and doxorubicin-alone treatment groups increased and remained at approximately 4-fold that of doxorubicin+eIF5A siRNAtreated H9c2 cells. In contrast, a slight change in the ROS level was found in cells transfected with doxorubicin+eIF5A siRNA and doxorubicin + metformin. These findings indicate that the production of eIF5A induced by doxorubicin may lead to $\mathrm{H} 9 \mathrm{c} 2$ cell apoptosis via the mitochondrial damage pathway.

Doxorubicin-Induced Loss of Mitochondrial Membrane Potential and Cytochrome-c Release in H9c2 Cells via eIF5A Mitochondrial permeability transition has been implicated in $\Delta \psi_{\mathrm{m}}$ collapse. We thus explored the role of the relative change in $\Delta \psi_{\mathrm{m}}$ in $\mathrm{H} 9 \mathrm{c} 2$ cells overexpressing eIF5A. The dye JC-1 was used to monitor $\Delta \psi_{\mathrm{m}}$ estimated as the 590-nm: 527-nm emission ratio. The $\Delta \psi_{\mathrm{m}}$ value of the doxorubicin-alone treatment group remained at low levels compared with the doxorubicin+eIF5A siRNA group and doxorubicin +metformin group (metformin can promote mitochondrial biosynthesis, as shown in Fig. 4A). Moreover, the 
A

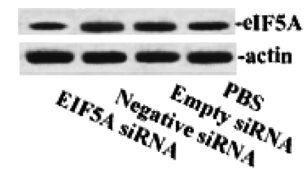

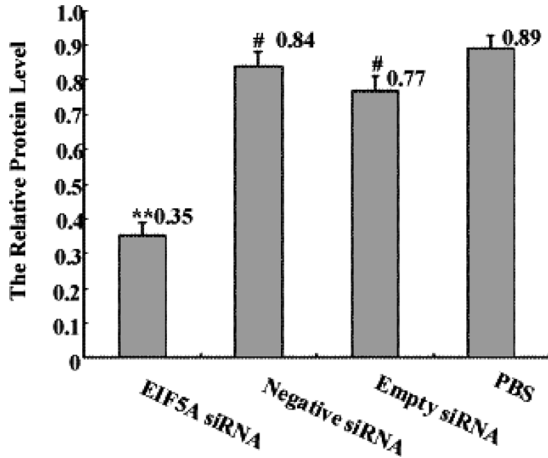

B
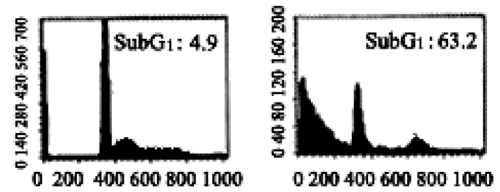

Doxorubicin+ eIF5A siRNA Doxorubicin+ empty vector

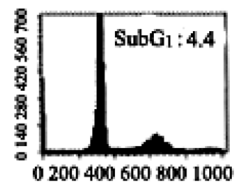

Doxorubicin+ Metformin

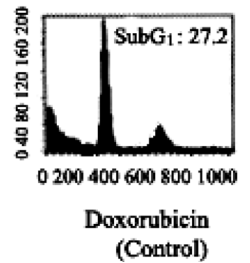

C

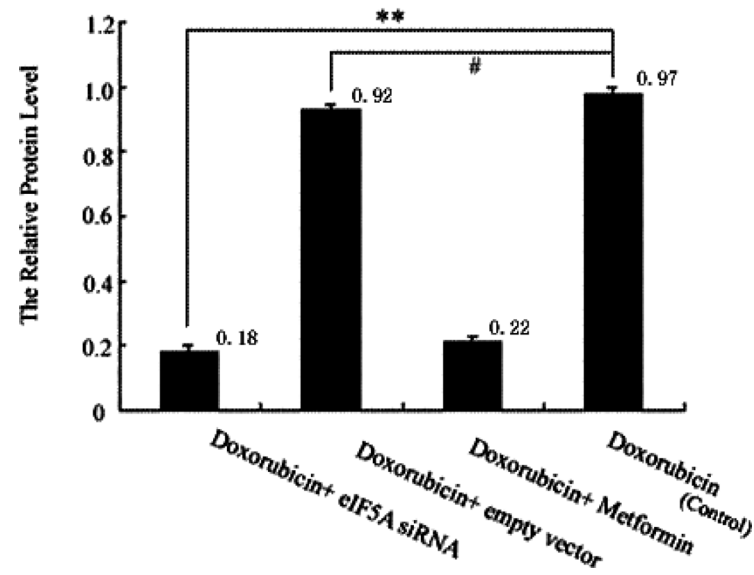

Fig. 2. eIF5A-Induced Apoptosis Death of H9c2 Cell

(A) Expression of eIF5A protein in H9c2 cardiomyocytes treated with siRNA was assessed in this experiment. The feasibility and effectiveness of siRNA on silencing target gene were detected using Western blot analysis. The results clearly showed the magnitude of low-expression (track 1) compared with negative siRNA, empty siRNA and PBS (tracks 2 4). The relative eIF5A protein levels were normalized to $\beta$-actin. These data are representative of three separate experiments, $* * p<0.01$ versus PBS group, $\# p>0.05$ versus $\mathrm{PBS}$ group. (B) H9c2 cells were treated with doxorubicin+eIF5A siRNA, doxorubicin + empty vector, doxorubicin + metformin (a promoter of mitochondrial biosynthesis, positive control), and doxorubicin alone (control). At $48 \mathrm{~h}$ posttransfection, cells were stimulated with doxorubicin $(2 \mu \mathrm{M})$. After further incubation for $18 \mathrm{~h}$, cells were subjected to FACS analysis as in "Experimental Procedures." (C) Effects of eIF5A on caspase-9 activation in H9c2 cells. H9c2 cells were exposed to doxorubicin+eIF5A siRNA, doxorubicin+empty vector, doxorubicin + metformin (positive control), and doxorubicin alone (control) for $48 \mathrm{~h}$. Cell were lysed and examined for the 17-kDa caspase-9 fragments using Western blotting. The relative caspase-9 protein levels were normalized to $\beta$-actin. $* * p<0.01$ versus doxorubicin group; $\# p>0.05$ versus doxorubicin group.

relative loss of membrane potential in the doxorubicin + eIF5A siRNA, doxorubicin+empty vector, doxorubicin+ metformin, and doxorubicin-alone groups was observed from $48 \mathrm{~h}$ of culture and continued until $72 \mathrm{~h}$. Thus changes in membrane potential in comparison with the control at a specific time period, i.e., $60 \mathrm{~h}$ of culture, was reduced. An approximately $50 \%$ loss in $\Delta \psi_{\mathrm{m}}$ was observed in the doxorubicin+empty vector group and doxorubicin-alone group compared with the doxorubicin+eIF5A siRNA group. The doxorubicin+eIF5A siRNA treatment of $\mathrm{H} 9 \mathrm{c} 2$ cells resulted in a $20 \%$ increase in $\Delta \psi_{\mathrm{m}}$ compared with the doxorubicin + metformin group after the initial manipulation (Fig. 4B). As seen in Fig. 4C, a release of cytochrome- $c$ into the cytosol was detected relative to a decrease in mitochondrial cytochrome- $c$ in the doxorubicin+empty vector and doxorubicin-alone group, whereas in the doxorubicin+eIF5A siRNA and doxorubicin + metformin groups, the expression of cytochrome- $c$ maintained the normal level. In the mitochondrial pathway, eIF5A induced by doxorubicin disrupts $\Delta \psi_{\mathrm{m}}$ and releases cytochrome-c into the cytoplasm of $\mathrm{H} 9 \mathrm{c} 2$ cells.
Effects of Doxorubicin on Phosphorylation of MAPKs in H9c2 Cells Figure 5 shows that phospho-p38 MAPK and phospho-JNK were observed after doxorubicin+eIF5A siRNA, doxorubicin+empty vector, doxorubicin+metformin, and doxorubicin-alone treatment for the specified times. The proteins of phospho-p38 MAPK and phospho-JNK were notably increased in the doxorubicin+empty vector and doxorubicin-alone treatment groups compared with the doxorubicin+eIF5A siRNA and doxorubicin+metformin groups. Because metformin can promote mitochondrial biosynthesis, the activation of phospho-p38 MAPK and phospho-JNK markedly decreased. This suggests that the expression of eIF5A induced by doxorubicin is related to the activation of the p38 MAPK and JNK signaling pathways.

SB202190 and SP600125 Reverse Doxorubicin-Induced H9c2 Cell Apoptosis In this experiment, we examined the role of the p38 MAPK and JNK pathways in H9c2 cell survival induced by doxorubicin. H9c2 cells were treated with SB202190 $10 \mu \mathrm{M}$ (p38 MAPK inhibitor) and SP600125 $10 \mu \mathrm{M}$ (JNK inhibitor) for $18 \mathrm{~h}$. As seen in Fig. 6A, a release 
A

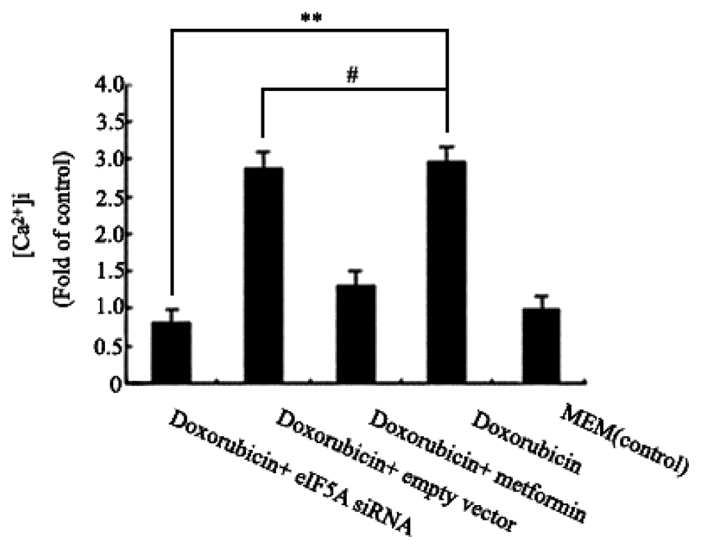

B

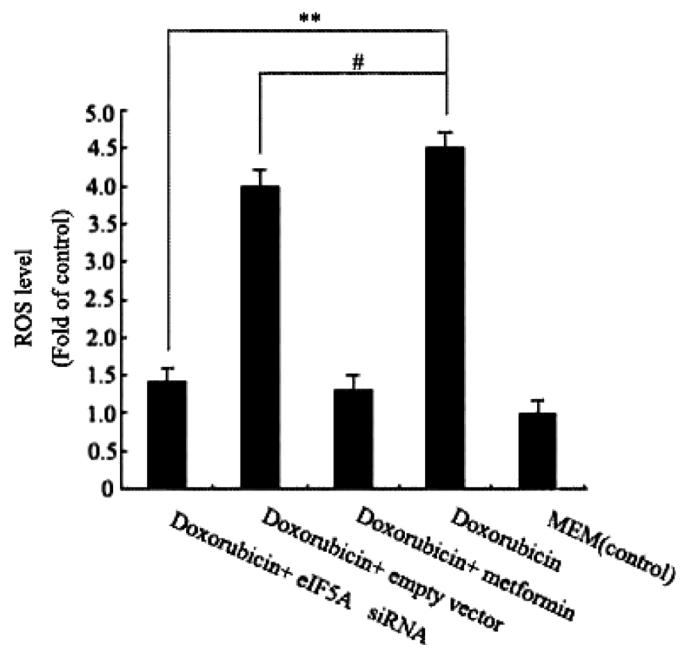

Fig. 3. eIF5A-Induced Increase in $\left[\mathrm{Ca}^{2+}\right]_{\mathrm{i}}$ and ROS Generation in $\mathrm{H} 9 \mathrm{c} 2 \mathrm{Cell}$

(A) eIF5A-elicited elevation of $\left[\mathrm{Ca}^{2+}\right]_{\text {: }}$. The intracellular $\mathrm{Ca}^{2+}$ concentration was quantified using a fluorescence plate reader after loading the cells with the calcium indicator (Fluo-4 AM). Results are shown as fold-increase in fluorescence intensity compared with the control group. The data represent mean \pm S.D. These data were representative of three separate experiments, $* * p<0.01$ versus doxorubicin group, $\# p>0.05$ versus doxorubicin group. (B) elF5A-induced ROS generation in H9c2 cells. After treatment with doxorubicin+eIF5A siRNA, doxorubicin+empty vector, doxorubicin + metformin (positive control), and doxorubicin alone (control) for the indicated time periods, ROS generation was quantified using a fluorescence plate reader. Results are shown as fold increase in fluorescence intensity compared with the control group. The data represent mean \pm S.D. These data were representative of three separate experiments, $* * p<0.01$ versus doxorubicin group, $\# p>0.05$ versus doxorubicin group.

A

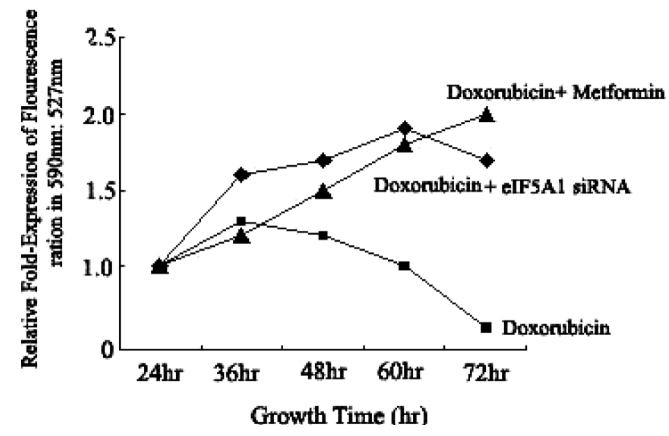

B

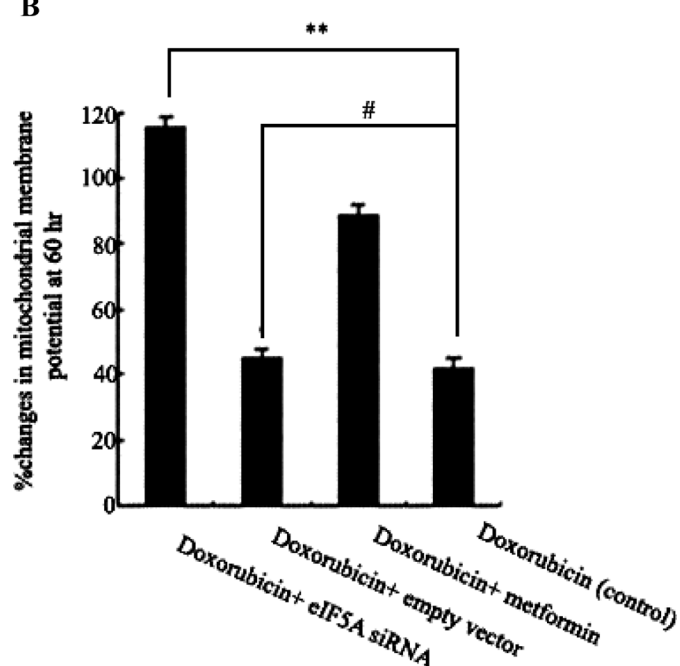

C

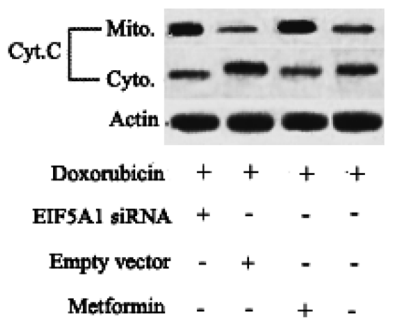

Cyt. $\mathrm{C}$ in Mito.

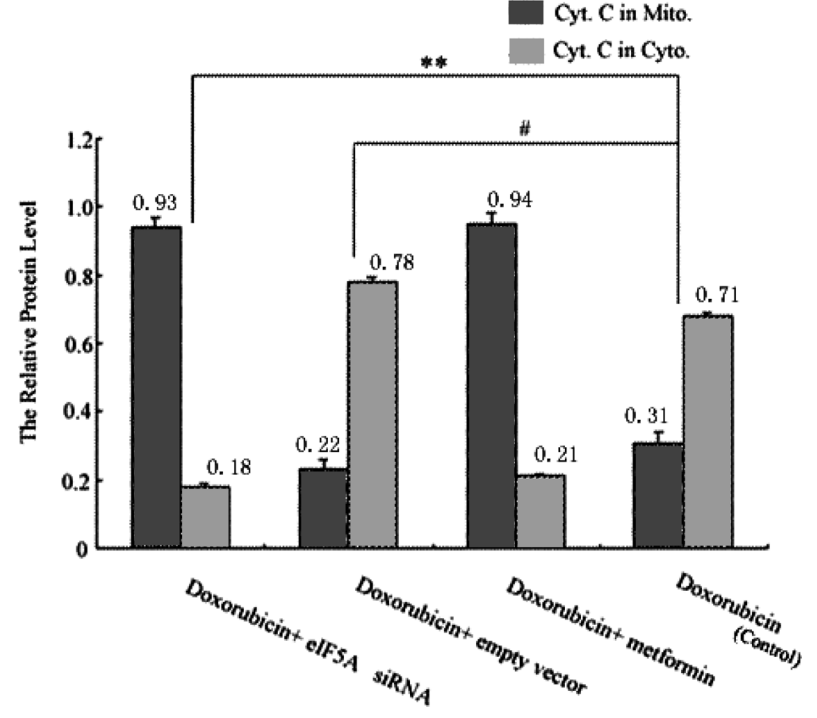

Fig. 4. Gradual Loss in Mitochondrial Membrane Potential Occurs in H9c2 Cell

(A) H9c2 cells were treated with eIF5A siRNA, empty vector, and MEM alone. At $48 \mathrm{~h}$ posttransfection, cells were stimulated with doxorubicin. After further incubation for $18 \mathrm{~h}$, time-dependent changes in the relative $\Delta \psi_{\mathrm{m}}$ value as detected by the fluorescence of JC-1 was expressed as the ratio of $590 \mathrm{~nm}$ (aggregated) to $527 \mathrm{~nm}$ (monomer). Data are mean \pm S.D. of three experiments. (B) When the change in relative $\Delta \psi_{\mathrm{m}}$, as detected by the fluorescence of JC-1 (590 nm:527 nm) in cells treated with doxorubicin + eIF $5 \mathrm{~A}$ siRNA, doxorubicin + empty vector, doxorubicin + metformin and doxorubicin alone at $60 \mathrm{~h}$ of growth was estimated. These data are representative of three separate experiments. $* * p<0.01$ versus doxorubicin group, $\# p>0.05$ versus doxorubicin group. (C) Western blot analyses of cytochrome-c in H9c2 cells treated with eIF5A siRNA, empty vector, and MEM alone. At $48 \mathrm{~h}$ posttransfection, cells were stimulated with doxorubicin. After further incubation for $18 \mathrm{~h}$, cells were subjected to Western blot analysis. The relative protein levels were normalized to $\beta$-actin. $* * p<0.01$ versus doxorubicin group; $\# p>0.05$ versus doxorubicin group. 


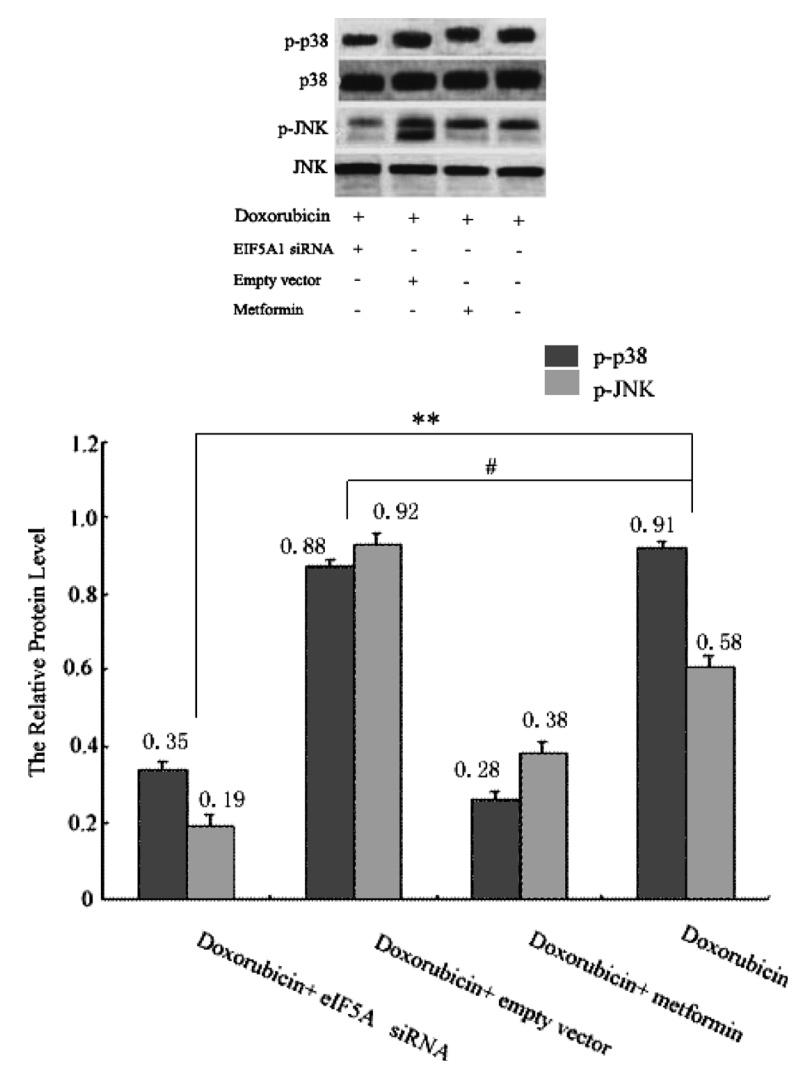

Fig. 5. Effects of eIF5A Treatment on Phosphorylation of MAPKs in H9c2 Cell

Western blot analyses of phospho-p38 MAPK and phospho-JNK in cells treated with doxorubicin+eIF5A siRNA, doxorubicin+empty vector, doxorubicin+metformin, and doxorubicin alone. At $48 \mathrm{~h}$ posttransfection, H9c2 cells were stimulated with doxorubicin $(2 \mu \mathrm{M})$. After further incubation for $18 \mathrm{~h}$, cells were subjected to Western blot analysis as in "Experimental Procedures." The relative protein level of p-p38 MAPK or $\mathrm{p}-\mathrm{JNK}$ was normalized to total $\mathrm{p} 38$ and JNK, respectively. $* * p<0.01$ versus doxorubicin group; $\# p>0.05$ versus doxorubicin group.

of cytochrome- $c$ into the cytosol was detected relative to a decrease in mitochondrial cytochrome- $c$ in doxorubicintreated $\mathrm{H} 9 \mathrm{c} 2$ cells. To evaluate caspase- 9 activation, the presence of the $17-\mathrm{kDa}$ caspase- 9 fragment was examined in $\mathrm{H} 9 \mathrm{c} 2$ cells by treating them with doxorubicin, doxorubicin+ SB202190 and doxorubicin+SP600125. Doxorubicin induced an increase in the $17-\mathrm{kDa}$ fragment during the 18 -h period, but the inhibitors SB202190 and SP600125 reversed the doxorubicin-induced release of cytochrome- $c$ and activation of caspase-9. As shown Fig. 6B, the number of cells in the subG1 region (H9c2 cell apoptosis) was significantly decreased in the doxorubicin+SB202190 and doxorubicin+SP600125 groups when compared with the doxorubicin-alone group in defined medium after $18 \mathrm{~h}$. This suggests that doxorubicininduced $\mathrm{H} 9 \mathrm{c} 2$ cell apoptosis is related to activation of the p38 MAPK and JNK signaling pathway.

\section{DISCUSSION}

It was reported that apoptosis is the principal process in doxorubicin-induced cardiomyopathy. ${ }^{14)}$ In this investigation, several typical apoptotic features, including mitochondrial dysfunction and caspase activation, were induced by doxorubicin in the H9c2 cardiacmyocyte cell line, which is used as a model for doxorubicin-induced cardiac toxicity. First, we demonstrated that the cardiacmyocyte apoptosis caused by doxorubicin was associated with the upregulation of eIF5A
A
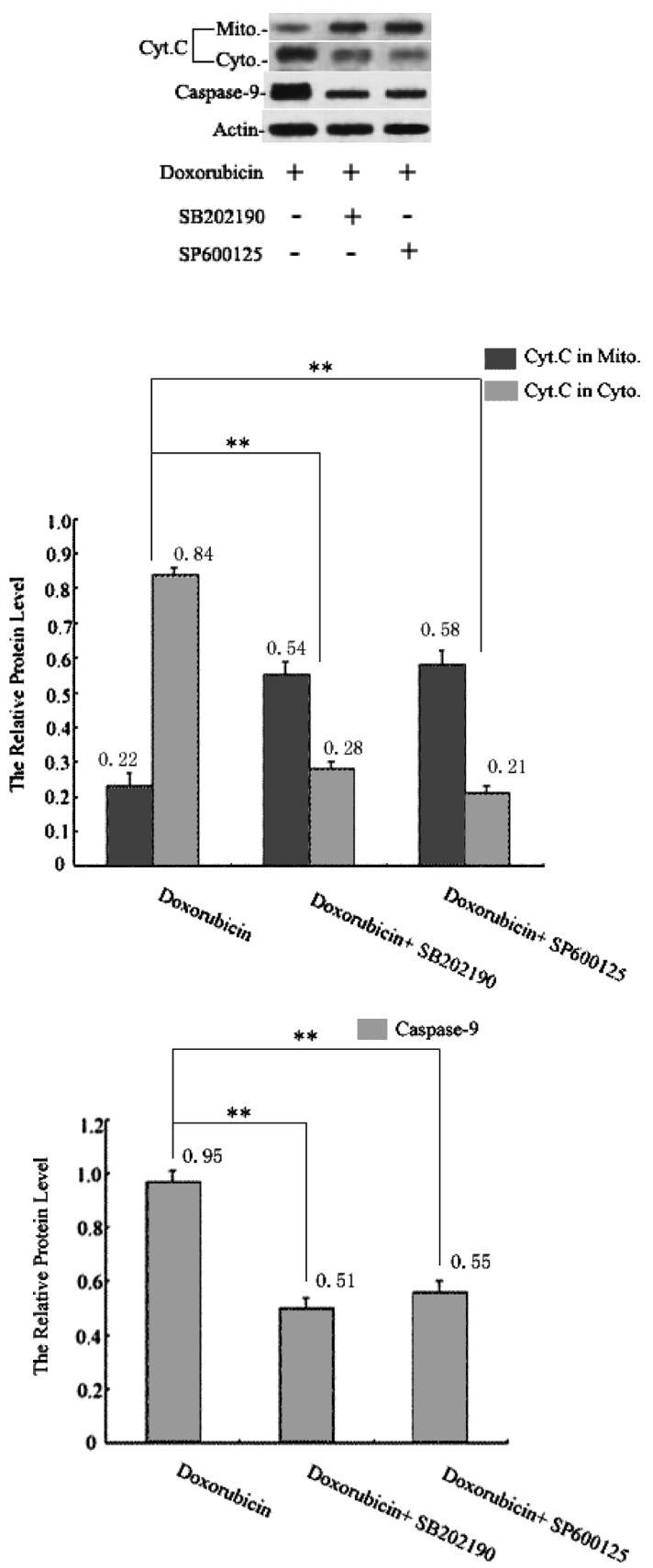

B
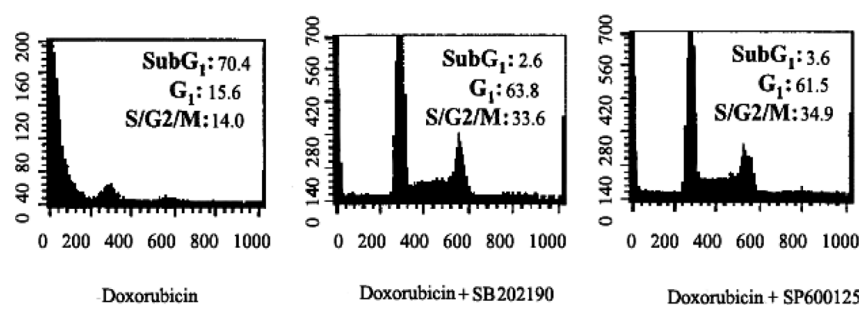

Fig. 6. Inhibitors SB202190 and SP600125 Reverse the DoxorubicinInduced $\mathrm{H} 9 \mathrm{c} 2$ Cell Apoptosis

(A) Effects of SB202190 and SP600125 on cytochrome- $c$ release and caspase-9 protein expression in doxorubicin-treated cells. H9c2 cells were treated with SB202190 $10 \mu \mathrm{M}$ (p38 MAPK inhibitor) and SP600125 $10 \mu \mathrm{M}$ (JNK inhibitor) for $18 \mathrm{~h}$, then incubated with doxorubicin $(2 \mu \mathrm{M})$. After further incubation for $18 \mathrm{~h}$, cells were subjected to Western blot analysis. Relative protein levels were normalized to $\beta$-actin. $* * p<0.01$ versus doxorubicin group. (B) H9c2 cells were treated with SB202190 $10 \mu \mathrm{M}$ and SP600125 $10 \mu \mathrm{M}$ for $18 \mathrm{~h}$, then incubated with doxorubicin. After further incubation for $18 \mathrm{~h}, \mathrm{H} 9 \mathrm{c} 2$ cells were subjected to FACS analysis as in Materials and Methods. 
protein (Fig. 1).

eIF5A is a multifunctional cellular protein expressed in a wide range of tissue and cell types including lymphocytes, endothelial cells, dendritic cells, and platelets. ${ }^{15)}$ Several studies also found a role for eIF5A in the regulation of apoptosis. Overexpression of eIF5A was found to induce apoptosis in hepatocellular ${ }^{16)}$ and colon ${ }^{17)}$ cancer cell lines. In our experiments, the stable overexpressing form of eIF5A was prepared by treating $\mathrm{H} 9 \mathrm{c} 2$ cells with doxorubicin. As shown in Fig. 2, flow cytometry analysis further revealed that the number of cells in the sub-G1 region increased after treatment with doxorubicin+empty vector and doxorubicin alone. Growth inhibition of $\mathrm{H} 9 \mathrm{c} 2$ cells was further characterized with the induction of apoptosis as indicated by caspase9 expression. The number of cells in the sub-G1 region and the expression of caspase- 9 both decreased after treatment with doxorubicin+eIF5A siRNA, and doxorubicin+metformin. These data indicate that doxorubicin may induce apoptosis of H9c2 cells via the effects of eIF5A protein.

Increasing evidence suggests that altered mitochondrial function is linked to apoptosis, and a decreasing mitochondrial transmembrane potential is associated with mitochondrial dysfunction. ROS are generated in mitochondria as a product of oxidative phosphorylation ${ }^{18)}$ and at low concentrations are involved in normal cell signaling. However, when ROS are generated in excess in defective mitochondria, they may act as a cytotoxic factor initiating apoptosis. ${ }^{19)}$ We found that the generation of ROS, creating mitochondrial dysfunction, led to apoptosis by doxorubicin-induced $\mathrm{H} 9 \mathrm{c} 2$ cells expressing eIF5A, a unique amino acid hypusine, following its accumulation in mitochondria.

Oxidant generation correlated with intracellular $\mathrm{Ca}^{2+} \mathrm{ac}-$ cumulation. This observation was also supported by the release of cytochrome- $c$ after transfecting H9c2 cells with doxorubicin+empty vector and doxorubicin alone. Meanwhile, we observed that doxorubicin+eIF5A siRNA mediated the reduction in eIF5A production in this transformant and conferred protection against ROS generation and apoptosis induction by decreasing caspase-9 expression in $\mathrm{H} 9 \mathrm{c} 2$ cells. It is speculated that eIF5A may regulate intramitochondrial concentrations of divalent cations such as $\mathrm{Ca}^{2+}$. It was expected that interference with electron transport by ROS and intracellular $\mathrm{Ca}^{2+}$ would influence $\Delta \psi_{\mathrm{m}}$. As expected, the changes in $\Delta \psi_{\mathrm{m}}$ in doxorubicin +empty vector- and doxorubicin-alone-expressing cells were observed as a consequence, thus inducing the apoptotic signaling pathway. All these characteristic features of eIF5A augment our present observations on mitochondrial $\mathrm{Ca}^{2+}$ overload in eIF5A overexpressing cells, suggesting its role in mitochondria-dependent apoptosis.

Since activated MAPKs are common components of the apoptotic program, the activated MAPK family members including p-p38 MAPK and p-JNK in cells treated with doxorubicin+eIF5A siRNA, doxorubicin+empty vector, doxorubicin + metformin, and doxorubicin alone were examined. Figure 5 shows that phospho-p38 MAPK and phospho-JNK were notably increased after doxorubicin+empty vector and doxorubicin-alone treatment for $30 \mathrm{~min}$. Alternatively, eIF5A siRNA treatment reduced the level of phospho-p38 MAPK and phospho-JNK. Pretreatment with SP600125 and/or SB202190 increased the survival of doxorubicin+empty vector and doxorubicin alone-treated H9c2 cells (Fig. 6B). This suggests that the activation of p38 MAPK and JNK was related to doxorubicin-induced apoptotic H9c2 cells death via the function of eIF5A.

As ROS are essential for triggering and completing apoptosis, our present results on the generation of ROS by eIF5A overexpression can be correlated with doxorubicin-induced $\mathrm{H} 9 \mathrm{c} 2$ cell apoptosis. It is a novel finding that the regulatory role of eIF5A in ROS generation causes mitochondrial dysfunction. However, eIF5A treatment initiates $\mathrm{Ca}^{2+}$ influx and then activates p38 MAPK and JNK, which modulate the expression of caspase-9. Then alterations in mitochondrial permeability and $\Delta \psi_{\mathrm{m}}$ loss trigger cytochrome-c release, caspase activation, and $\mathrm{H} 9 \mathrm{c} 2$ cell apoptosis. We suggest that eIF5A could play an important role in $\mathrm{H} 9 \mathrm{c} 2$ cell growth promotion or growth inhibition depending on its level of expression and subcellular context.

Acknowledgments This study was supported in part by Nanjing Medical University Science and the Technology Development Foundation (No. NJMUM029).

\section{REFERENCES}

1) Grange C., Geninatti-Crich S., Esposito G., Alberti D., Tei L., Bussolati B., Aime S., Camussi G., Cancer Res., 70, 2180-2190 (2010).

2) Miyagawa K., Emoto N., Widyantoro B., Nakayama K., Yagi K., Rikitake Y., Suzuki T., Hirata K., Hypertension, 55, 738-746 (2010).

3) Bien S., Riad A., Ritter C. A., Gratz M., Olshausen F., Westermann D., Grube M., Krieg T., Ciecholewski S., Felix S. B., Staudt A., Schultheiss H. P., Ewert R., Völker U., Tschöpe C., Kroemer H. K., Cancer Res., 67, 10428-10435 (2007)

4) Riad A., Bien S., Westermann D., Becher P. M., Loya K., Landmesser U., Kroemer H. K., Schultheiss H. P., Tschöpe C., Cancer Res., 69, 695-699 (2009).

5) Shizukuda Y., Matoba S., Mian O. Y., Nguyen T., Hwang P. M., Mol. Cell. Biochem., 273, 25-32 (2005).

6) Esaki M., Takemura G., Kosai K., Takahashi T., Miyata S., Li L., Goto K., Maruyama R., Okada H., Kanamori H., Ogino A., Ushikoshi H., Minatoguchi S., Fujiwara T., Fujiwara H., Am. J. Physiol. Heart Circ. Physiol., 294, H1048-H1057 (2008)

7) Li L., Takemura G., Li Y., Miyata S., Esaki M., Okada H., Kanamori H., Ogino A., Maruyama R., Nakagawa M., Minatoguchi S., Fujiwara T., Fujiwara H., Lab. Invest., 87, 440-455 (2007).

8) Dias C. A., Cano V. S., Rangel S. M., Apponi L. H., Frigieri M. C., Muniz J. R., Garcia W., Park M. H., Garratt R. C., Zanelli C. F., Valentini S. R., FEBS J., 275, 1874-1888 (2008).

9) Sun Z., Cheng Z., Taylor C. A., McConkey B. J., Thompson J. E., J. Cell. Physiol., 223, 798-809 (2010).

10) Costa-Neto C. M., Parreiras-e-Silva L. T., Ruller R., Oliveire E. B., Miranda A., Oliveire L., Ward R. J., Biochem. Biophys. Res. Commun., 347, $634-640$ (2006).

11) Zou M. H., Kirkpatrick S. S., Davis B. J., Nelson J. S., Wiles W. G. IV Schlattner U., Neumann D., Brownlee M., Freeman M. B., Goldman M. H., J. Biol. Chem., 279, 43940-43951 (2004).

12) Meenakshi J., Anupama S. K., Datta K., Biochem. Biophys. Res. Commun., 300, 686-693 (2003).

13) Dey R., Moraes C. T., J. Biol. Chem., 275, 7087-7094 (2000)

14) Li L., Takemura G., Li Y., Miyata S., Esaki M., Okada H., Kanamori H., Khai N. C., Maruyama R., Ogino A., Minatoguchi S., Fujiwara T., Fujiwara H., Circulation, 113, 535-543 (2006).

15) Gentz P. M., Blatch G. L., Dorrington R. A., FEBS J., 276, 695-706 (2009).

16) Tang D. J., Dong S. S., Ma N. F., Xie D., Chen L., Fu L., Lau S. H., Li Y., Li Y., Guan X. Y., Hepatology, 51, 1255-1263 (2010).

17) Taylor C. A., Sun Z., Cliche D. O., Ming H., Eshaque B., Jin S., Hopkins M. T., Thai B., Thompson J. E., Exp. Cell. Res., 313, 437-449 (2007).

18) Dey R., Moraes C. T., J. Biol. Chem., 275, 7087-7094 (2000).

19) Green D. R., Reed J. C., Science, 281, 1309-1312 (1998). 